\title{
Evaluation of human fetal bone implants in SCID mice as a model of prostate cancer bone metastasis
}

\author{
ARUN S. SINGH ${ }^{1,2}$, GORDON R. MaCPHERSON ${ }^{1}$, DOUGLAS K. PRICE ${ }^{1}$, \\ DANIEL SCHIMEL ${ }^{3}$ and WILLIAM D. FIGG ${ }^{1}$ \\ ${ }^{1}$ Medical Oncology Branch, Center for Cancer Research, National Cancer Institute, Bethesda, MD 20892; \\ ${ }^{2}$ Howard Hughes Medical Institute, Chevy Chase, MD 20815; ${ }^{3}$ Mouse Imaging Facility, \\ Center for Cancer Research, National Cancer Institute, Bethesda, MD 20892, USA
}

Received June 27, 2005; Accepted July 22, 2005

\begin{abstract}
The metastasis of prostate cancer cells to the bone marrow constitutes the major source of morbidity and mortality in prostate cancer. Studying this process has been hampered by the lack of preclinical models to evaluate novel therapeutics and to study the biology of the disease. One proposed model utilizes human fetal bone implants to serve as the target for prostate cancer cells injected via the tail vein. We employed this model to test the ability of zoledronic acid to prophylax and to treat bone metastases. To improve the rate of bone metastasis, we used two bone implants instead of one to evaluate the cell lines PC3 and PC $3 \mathrm{M}$, a more metastatic subline. For this purpose we generated the novel cell line PC3EGFPLuc, which can be used for luminescence and/or fluorescence imaging in vivo. We did not observe bone implant metastases in 52 mice, with 90 bone implants following tail vein injection of $1 \times 10^{6} \mathrm{PC} 3$ or PC $3 \mathrm{M}$ cells. Soft tissue lesions in the buttocks and hind limbs as well as cellular growth in the hindlimbs were observed via bioluminescence imaging. This evidence together with literature findings suggests that this model produces artifactual 'bone metastasis' lesions.
\end{abstract}

\section{Introduction}

Metastasis to bone is the major cause of morbidity and mortality in prostate cancer patients. Of the 232000 men who developed prostate cancer in 2004, approximately 30000 died secondary to metastasis (1). Clinically, patients with prostate cancer bone metastasis are treated with hormone ablation

Correspondence to: Dr William Douglas Figg, Medical Oncology Branch, Center for Cancer Research, National Cancer Institute, Bldg. 10/Room 5A01 MSC 1910, 9000 Rockville Pike, Bethesda, MD 20892, USA

E-mail: wdfigg@helix.nih.gov

Key words: prostate, cancer, metastasis, bone, imaging, luminescence, $\mathrm{PC} 3$ and/or radiation therapy. However, these regimens do not improve life expectancy. Recently, two phase III trials showed that docetaxel with prednisone or estramustine significantly increased life expectancy by about 2 months $(2,3)$. In addition, the bisphosphonate zoledronate was shown to improve bone pain and skeletal-related events in men with prostate cancer bone metastasis (4).

Prostate cancer bone metastases are predominantly osteoblastic lesions admixed with areas of osteolysis (5). Pathological studies have shown that early stage bone metastases tend to be more osteolytic, while later stages shift towards a predominant osteoblastic phenotype (6-9). These observations are supported by the finding that osteolysis may precede the osteoblastic pathology (10). Hence, investigators have targeted the osteolytic process in attempts to prevent prostate cancer bone metastasis (11-13).

The development of efficacious therapeutics for bone metastases has been hampered by the lack of preclinical models to study its biology. The most commonly used models involve either intravenous, intracardiac, orthotopic or intratibial injection of prostate cancer cells. In addition, immunodeficient mice transplanted with human bone fragments and transgenic mice are being actively pursued as alternative models. One recent study introduced the SCID-hu (severe combined immunodeficient-human) model for studying prostate cancer bone metastasis (14). In this model, human fetal bone fragments are subcutaneously implanted into SCID mice to serve as a target for prostate cancer cells injected via the tail vein.

The bisphosphonate zoledronate has been used to prophylax and treat prostate cancer bone lesions. The models used in the evaluation of this drug have involved the injection of prostate cancer cells into the tibias of SCID mice and thus do not reflect the multistep nature of metastasis $(11,12)$. In this study, we evaluated the ability of zoledronate to prophylax and treat prostate cancer bone metastasis using the SCID-hu system as our model of metastasis. This model is advantageous because it recreates many of the stages of the metastatic process, produces osteolytic lesions and is tissue- and species-specific $(5,14)$. Its major disadvantage is that it yields low rates of metastases ( 26\%) (14). 


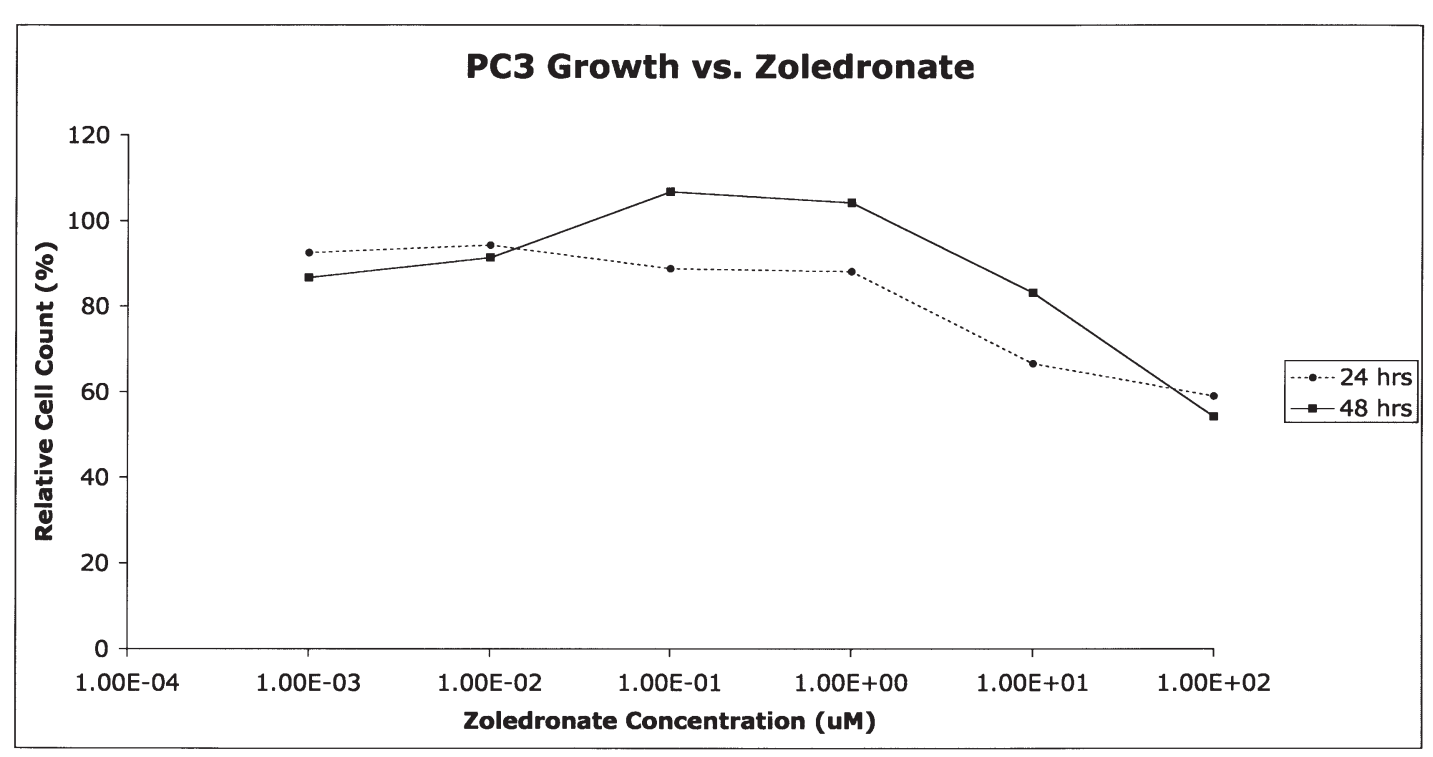

Figure 1. Effects of zoledronate on PC3 cell growth. PC3 cells were incubated with various concentrations of zoledronate for 24 or $48 \mathrm{~h}$. At the end of each time point, cells were trypsinzed and counted.

\section{Materials and methods}

Cell culture, transfection and subcloning. The prostate cancer cell lines PC3 (American Type Culture Collection, Manassas, VA) and PC3M (gift from Dr I. Fidler) cells were maintained at $37^{\circ} \mathrm{C}$ and $5 \% \mathrm{CO}_{2}$ in RPMI-1640 supplemented with $10 \%$ heat-inactivated FBS, penicillin (100 units $/ \mathrm{ml}) /$ streptomycin $(100 \mu \mathrm{g} / \mathrm{ml})$ and amphotericin B $(0.25 \mu \mathrm{g} / \mathrm{ml})$.

The pEGFPLuc plasmid was purchased from Clontech (Palo Alto, CA). PC3 cells $\left(3 \times 10^{4}\right)$ were stably transfected with the pEGFPLuc plasmid using the SuperFect transfection reagent (Qiagen, Valencia, CA) and selected with $200 \mu \mathrm{g} / \mathrm{ml}$ G418 (Invitrogen). This concentration was derived from determining the kill curve for the cell lines being transfected. Cells used for injection were monitored by fluorescence microscopy and checked for luminescence using a luciferase reporter kit (Clontech, Palo Alto, CA). The lower limit of detection of the transfected cell lines was determined by serially diluting 10000 PC3EGFPLuc cells and quantified on the IVIS system (Xenogen, Alameda, CA) 2 min after adding $100 \mu \mathrm{l}$ of $150 \mu \mathrm{g} / \mathrm{ml}$ D-Luciferin.

Bone implantation into mice. All animal experiments were approved by the Animal Care and Use Committee of the National Institutes of Health. SCID mice were maintained in pathogen-free housing.

Six- to eight-week-old male SCID mice (Taconic Farms, NY) were anesthetized with an inducing mixture of 5\% isoflurane mixed with nitrogen and oxygen and maintained on $2 \%$ isoflurane during the procedure. The lower backs of SCID mice were shaved and sanitized and $0.5 \mathrm{~cm}$ incisions were made. Sixteen- to eighteen-week-old human fetal bone was obtained from Advanced Bioscience Resources (Alameda, $\mathrm{CA}$ ) and cut into $0.5 \mathrm{~cm}$ sections and split along the long axis. This tissue was subject to one freeze-thaw cycle. Two pieces of bone were subcutaneously implanted into each mouse, with the marrow cavity placed adjacent to the mouse muscle, and the skin was closed with three to five $4-0$ chromic sutures. The
Table I. PC3M metastasis to mouse organs.

Pathology PC3M (n=12)

Human bone

$0 / 7^{\mathrm{a}}$

implant metastasis

Mouse bone

marrow metastasis

Mouse bone

metastasis

Lung metastasis

2/12 femur

3/12 femur/vertebrae

2/12 had emboli

Lymph node

$4 / 12$

metastasis

Skin metastasis

2/10 (large tumors around buttocks)

Other

1 Lymphoblastic lymphoma 2/2 Pleural cavity metastasis

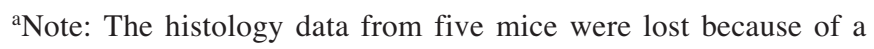
histopathology processing error.

viability of the bone implant was assessed histologically after 35 days.

In vitro effect of zoledronate on PC3 and PC3M. Cells were seeded in 12-well plates (Corning Costar, Acton, MA) in RPMI containing $10 \%$ FBS and antibiotics as above. At time of replating, zoledronate (Novartis) was dissolved in PBS and added to each well so that the final concentration was 1,10 or $100 \mu \mathrm{M}$. At 24 and $48 \mathrm{~h}$, the cells were trypsinized and counted. Cell growth was plotted against the log of the zoledronate concentration. 
Table II. PC3EGFPLuc metastasis to mouse organs.

\begin{tabular}{lccc}
\hline & Bone metastases (2 bones/mouse) & Lung metastases & Lymph node metastases \\
\hline Control & $0 / 8$ & $1 / 8$ & $0 / 7$ \\
Low-dose prophylaxis & $0 / 7$ & $0 / 7$ & $0 / 7$ \\
High-dose prophylaxis & $0 / 7$ & $0 / 7$ & $0 / 7$ \\
Low-dose treatment & $0 / 7$ & $1 / 7$ & $0 / 4$ \\
High-dose treatment & $0 / 7$ & $1 / 8$ & $0 / 6$ \\
\hline
\end{tabular}

Cell injection and zoledronate administration. Bone implants were allowed to graft for 35 days (14). At this time, PC3EGFPLuc or PC3M cells were introduced via tail vein injection. PC3EGFPLuc $\left(1 \times 10^{6}\right)$ or PC3M $\left(10^{5}\right)$ cells in RPMI were injected using 26 gauge needles. The animals injected with the PC3M cells were not treated with zoledronate. The mice injected with PC3EGFPLuc cells were subdivided into 5 groups. A control group consisted of 8 mice. The prophylactic arm of the study was subdivided into two groups: high-dose and low-dose prophylaxis groups, each starting with 8 mice. The low-dose and the high-dose groups received 15 and $50 \mu \mathrm{g}$ of zoledronate one day before injection of PC3EGFPLuc cells via subcutaneous administration with a 26 gauge needle in the nuchal area. Zoledronate was warmed to room temperature and was at $\mathrm{pH}$ 5.5-6.5 before injection. There were also 2 treatment groups: a high- and a low-dose group. In the treatment groups, zoledronate was given at weeks 2 and 4 in a manner analogous to the prophylaxis groups. The low-dose group received $15 \mu \mathrm{g}$ zoledronate, while the high-dose group received $50 \mu \mathrm{g}$.

Imaging. Mice were imaged for bioluminescence weekly from weeks 3 to 6 using the IVIS ${ }^{\circledR}$ imaging system from Xenogen (Alameda, CA). Image acquisition and processing was accomplished using the Living Image ${ }^{\circledR}$ software. Anesthesia was induced with ketamine/xylazine $(0.1 \mathrm{ml}$ of a cocktail made of $100 \mathrm{mg} / \mathrm{ml} \mathrm{ketamine} \mathrm{and} 10 \mathrm{mg} / \mathrm{ml}$ xylazine) administered intraperitoneally and maintained with $2 \%$ isoflurane mixed with oxygen/nitrogen via nosecone. Luciferin (3 mg dissolved in water) was given via i.p. injections and optical imaging was carried out approximately 5-10 min later. Three to five mice were imaged at a time. Tail, feet and paws were taped to prevent movement artifacts. One minute acquisitions were carried out under the high sensitivity setting of the instrument software. Background subtraction was accomplished using an automated feature of the program.

Histology. All bone implants were decalcified and analyzed for the presence of metastases. Necropsy was carried out for each animal and all gross lesions were stained with hematoxylin and eosin (H\&E) by a veterinary pathologist.

\section{Results}

In vitro growth inhibition of PC3 and PC3M cells following treatment with zoledronate. Zoledronate at $100 \mu \mathrm{M}$ inhibited PC3 cell growth by approximately $30 \%$ after 24 or $48 \mathrm{~h}$ of treatment. Concentrations of $\leq 1 \mu \mathrm{M}$ did not inhibit cell growth
(Fig. 1). Similar results were observed for the PC3M cell line under similar conditions.

PC3-EGFPLuc luminescence quantification. The PC3EGFPLuc cell line was found to have a lower limit of detection of approximately 78 cells in vitro as determined from a serial dilution assay (Fig. 2).

Bone metastasis experiments. The capacity of the human fetal bone fragments to engraft into the SCID mice was evaluated. One month post implantation, human bone implants were sent for histology to assess the viability of the bones. The bones were well vascularized and only a few small areas of necrosis were present (Fig. 3A).

In the first experiment, 12 mice were injected with $\mathrm{PC} 3 \mathrm{M}$ cells. Of the seven evaluable mice (five were lost due to histopathological error), none developed metastasis to the human bone implant. These bone implants were also vascularized and had sparse areas of necrosis analogous to the pilot samples. Three of twelve mice developed metastases to their vertebrae/ femurs, 1/10 developed metastases to its bone marrow, 3/12 developed metastases to the lungs and 4/12 developed lymph node metastases. Two of twelve also developed tumor growth in the skin and peritoneum. Two mice had emboli in their lungs in addition to the metastases present (Table I). Two of the mice developed large tumor growths in their hind regions; one developed an approximately $1 \mathrm{~cm}$ tumor lateral to the tail and the other developed about a $0.5 \mathrm{~cm}$ lesion around the area of his femur which affected his ability to move.

In a second trial, the PC3EGFPLuc cells did not metastasize to the human bone implants in the control, prophylaxis or treatment groups at six weeks after injection. Sparse lung metastases were present, but not enough to draw any conclusions about zoledronate's effects. Emboli (not tabulated) were also present. Lymph node metastases to the axillary and inguinal lymph nodes were not present (Table II). This cell line did not produce metastases to the mouse skeleton, as assessed by hematoxylin and eosin staining.

During the experiment some mice had to be sacrificed as per animal ethics. One mouse in the control group was sacrificed because of lethargy; no bone, lymph node or lung metastases were found in this mouse. In the high-dose treatment group, one mouse had to be sacrificed because of a necrotic tail on day 29; this mouse is included in Table II. Two mice from the low-dose treatment group were sacrificed on day 29 because of tail necrosis; these mice are also included in Table II. One mouse from the high-dose prophylaxis group was found dead on day 11 and was excluded from the study. One 


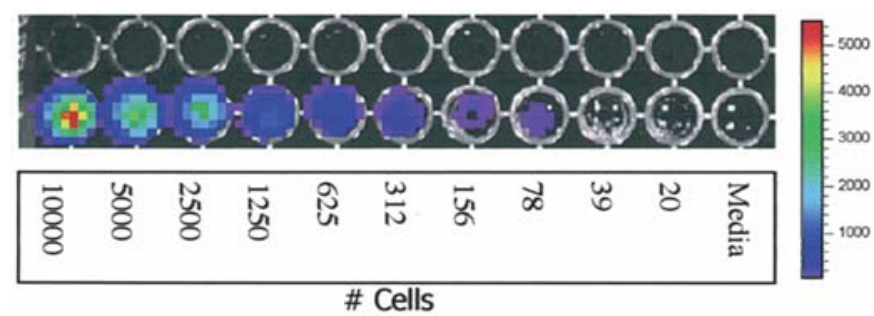

Figure 2. Lower limit of luminescence detection. Serially diluted PC3EGFPLuc cells were assayed for luminescence in a 96-well plate. This experiment was repeated in duplicate. Signal could be obtained from as few as 78 cells.

A

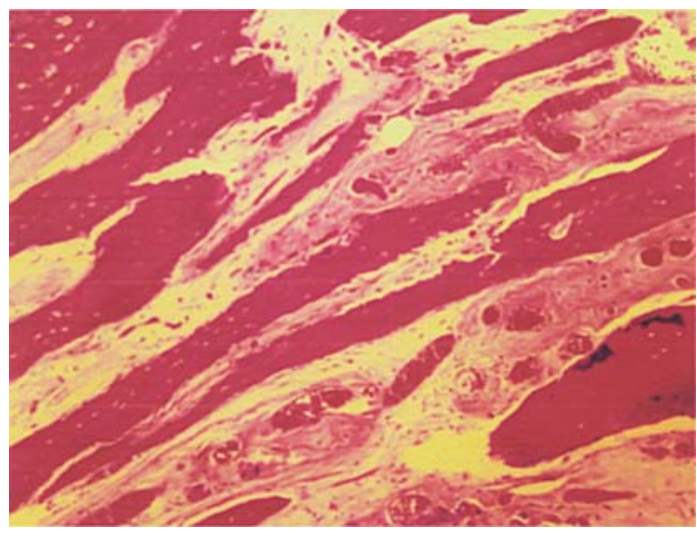

B

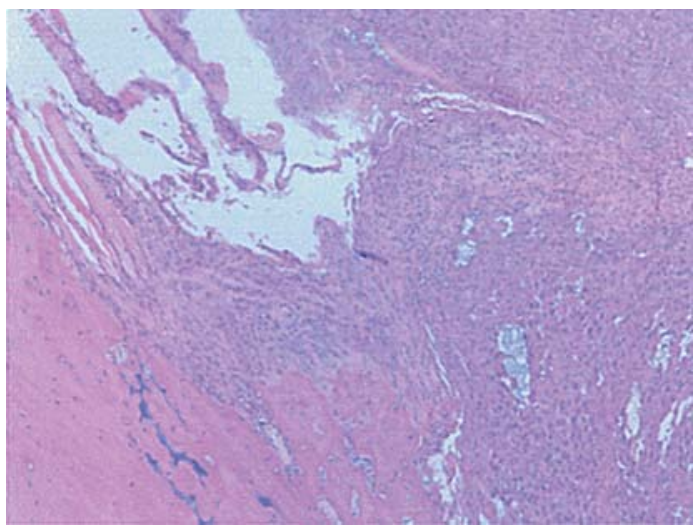

Figure 3. Histology. (A) Vascularized implanted bone showing normal bone architecture and vascularity. (B) PC3M cells metastasized to the mouse femur. Nests of cells with brightly staining nuclei are present.

mouse was found dead on day 41; this mouse is included in Table II. One mouse from the low-dose prophylaxis group had severe tail necrosis and was sacrificed; this mouse was excluded from the study.

Imaging metastases. Luminescence was not detected in the region of the bone implants in any of the mice despite weekly imaging from weeks 3-6. Weak signals were detected in

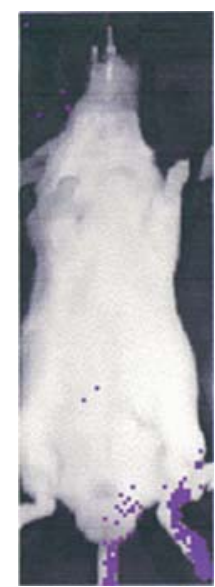

Figure 4. Example of bioluminescent imaging at week 4. Mice injected with PC3EGFPLuc cells showing luminescent signal from the foot of a mouse at week 4.

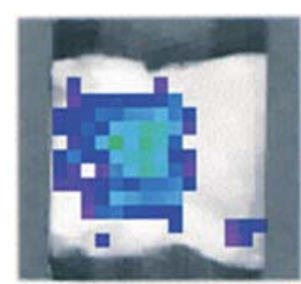

Figure 5. Example of bioluminescent imaging at week 6. Positive luminescent signal from the chest of a mouse from the PC3EGFPLuc control group at week 6 . This mouse had a pleural metastasis on histology. The lower body of the mouse is shielded to maximize signal ratio readings from the lungs.

hindlimbs and the thorax of some mice (Fig. 4). Of the three mice in which histologically confirmed lung metastases were present, a strong luminescence signal was obtained from one mouse, which had a pleuritic metastasis (Fig. 5). The two other mice with lung metastases did not produce strong luminescence signals.

\section{Discussion}

Animal models of prostate cancer bone metastasis are inefficient. One promising model proposed that human fetal bone implants in the backs of SCID mice could produce tissueand species-specific bone metastasis. However, the models produced a low rate of metastasis to the implanted bone fragments. To address this issue, we implanted two pieces of bone into each animal instead of one and evaluated the ability of the PC3M cell line, a more metastatic subclone of PC3, to metastasize (15). Furthermore, PC3 cells were labeled with luciferase in order to longitudinally follow the development of bone metastasis and to monitor the response of any tumor burden to zoledronate. This model appears to be variable and inefficient in reproducing bone metastasis, thus, its use is not appropriate for drug evaluation.

In our hands, the model was unsuccessful in establishing metastases to the human bone implants. Neither the PC3M cell 
line nor the PC3EGFPLuc cells resulted in metastases to the bone implants. We did not detect any metastases to the human bone implants histologically or by bioluminescence imaging. The PC3M cell line was initially selected because subclones have been reported to metastasize to bone at rates as high as $80 \%$ by intravenous injection and also to the legs of mice via intracardiac injections $(16,17)$. While this line did not produce lesions in the bone implants, it did produce metastases to various soft tissues as well as to mouse femur and vertebrae (Fig. 3B). In addition, it has been reported that stably tranfecting PC $3 \mathrm{M}$ cells with luciferase containing vectors also produce bone metastases at high rates, and thus, reduces the likelihood of transfection per se negatively influencing the metastatic ability (16).

The transfected PC3EGFPLuc cell line was assayed to determine the lower limit of detection of its luminescence. Around 78 cells could be detected via serial dilution assay, consistent with the $\mathrm{PC} 3 \mathrm{M}$ prostate cancer cell line transfected with luciferase reported in the literature (6). This is an important consideration in terms of estimating the smallest lesions from which a signal can be obtained.

In accordance with the report by Nemeth et al, we found that the fetal bone implants were well vascularized and viable, eliminating this as a source of error (Fig. 3A) (14). We evaluated the effects of zoledronate on in vitro cell viability. Treating cells with $\leq 1 \mu \mathrm{M}$ of zoledronate did not affect cell survival (Fig. 1). A concentration of $100 \mu \mathrm{M}$ was required before toxicity was observed. However, doses in this range were not used in the animal experiment. These doses were selected because they were of intermediate value between the dosages used in two previous studies using zoledronate for treating bone lesions which employed as little as $5 \mu \mathrm{g}$ of zoledronate to as much as $150 \mu \mathrm{g}(11,12)$. Human subjects typically receive around $57 \mu \mathrm{g} / \mathrm{kg}$ for a $70 \mathrm{~kg}$ person IV q3-4 weeks of zoledronate for bone lesions. The doses employed in our study were about 15-35 times higher than those employed in the recommended human studies; however, zoledronate was given by subcutaneous injection, not intravenous injection as in human studies.

We also did not observe a noticeable difference between treating cells for 24 or $48 \mathrm{~h}$. These findings make it unlikely that the drug treatment could have resulted in the absence of metastases in the experiments. In addition, the PC3M group and the PC3EGFPLuc control groups were not treated with zoledronate, but nevertheless did not produce metastases. Furthermore, the presence of metastases in the PC3M group and the visualization of luminescence from the PC3EGFPLuc group indicate that the cell lines used were viable and that the tail vein injections were executed properly.

While numerous hypotheses may explain these results, a few findings from these experiments shed light on the inability to produce metastases. In two out of twelve cases, we found that the PC $3 \mathrm{M}$ cell line formed hind limb tumors and tumors in the region adjacent to the buttock/hindlimb. In the luminescence visualization assays, it was noted that there were instances where a signal was found coming from the legs of the mice. These findings led us to propose that cells injected via the tail vein are likely to flow to these areas (Fig. 4). Furthermore, cells lodged in this region may have been stimulated to grow by the bone fragments implanted in the hindlimbs, thus mimicking metastasis, and may account for the observations by Nemeth et al (14). This phenomenon has been demonstrated before by seeding PC3 cells adjacent to human bone fragments (18). This may explain why Nemeth et al were able to find 'metastasis' in the bones implanted in the hindlimb, but not in the soft tissue organs implanted onto the lower backs of the mice. Furthermore, it was observed that isolating the 'bone metastases', subculturing them and repeating the experiment resulted in zero metastases (14). Typically, one would expect the metastasis rate to improve in such conditions $(15,19)$.

It may also be argued that our bones were in the lower back rather than the hindlimb, thus they were not subject to the same rate of blood flow. However, these two regions were not significantly distant from one another. Both bone implants were laid out onto muscle in our experiment and in the one by Nemeth et al (14). In conclusion, the inability of two different cells lines to produce any metastases in 52 mice, and $>90$ pieces of human fetal bone implant, may indicate the inconsistency of this model in producing metastases.

\section{Acknowledgements}

Hemotoxylin and eosin staining was done by the Laboratory Animal Sciences Program of the National Cancer Institute in Frederick, MD. We would like to thank Miriam Anver and Keith Rogers for assistance with histopathology. We would like to thank Eric Davis, Louis Staudt, Steve Libutti and Sandra O'Connor for help with instrumentation. This research was supported by the Intramural Research Program of the $\mathrm{NIH}$, National Cancer Institute, Center for Cancer Research.

\section{References}

1. Jemal A, Murray T, Ward E, Samuels A, Tiwari RC, Ghafoor A, Feuer EJ and Thun MJ: Cancer statistics. CA Cancer J Clin 55: 10-30, 2005

2. Petrylak DP, Tangen CM, Hussain MH, Lara PN Jr, Jones JA, Taplin ME, Burch PA, Berry D, Moinpour C, Kohli M, Benson MC, Small EJ, Raghavan D and Crawford ED: Docetaxel and estramustine compared with mitoxantrone and prednisone for advanced refractory prostate cancer. N Engl J Med 351: 1513-1520, 2004.

3. Tannock IF, de Wit R, Berry WR, Horti J, Pluzanska A, Chi KN, Oudard S, Theodore C, James ND, Turesson I, Rosenthal MA and Eisenberger MA: Docetaxel plus prednisone or mitoxantrone plus prednisone for advanced prostate cancer. N Engl J Med 351: 1502-1512, 2004.

4. Saad F, Gleason DM, Murray R, Tchekmedyian S, Venner P, Lacombe L, Chin JL, Vinholes JJ, Goas JA and Chen B: A randomized, placebo-controlled trial of zoledronic acid in patients with hormone-refractory metastatic prostate carcinoma. J Natl Cancer Inst 94: 1458-1468, 2002.

5. Mundy GR: Metastasis to bone: causes, consequences and therapeutic opportunities. Nat Rev Cancer 2: 584-593, 2002.

6. Charhon SA, Chapuy MC, Delvin EE, Valentin-Opran A, Edouard CM and Meunier PJ: Histomorphometric analysis of sclerotic bone metastases from prostatic carcinoma special reference to osteomalacia. Cancer 51: 918-924, 1983.

7. Galasko CS: Mechanisms of lytic and blastic metastatic disease of bone. Clin Orthop 169: 20-27, 1982.

8. Koutsilieris M: Osteoblastic metastasis in advanced prostate cancer. Anticancer Res 13: 443-449, 1993.

9. Urwin GH, Percival RC, Harris S, Beneton MN, Williams JL and Kanis JA: Generalised increase in bone resorption in carcinoma of the prostate. Br J Urol 57: 721-723, 1985.

10. Yi B, Williams PJ, Niewolna M, Wang Y and Yoneda T: Tumorderived platelet-derived growth factor-BB plays a critical role in osteosclerotic bone metastasis in an animal model of human breast cancer. Cancer Res 62: 917-923, 2002. 
11. Corey E, Brown LG, Quinn JE, Poot M, Roudier MP, Higano CS and Vessella RL: Zoledronic acid exhibits inhibitory effects on osteoblastic and osteolytic metastases of prostate cancer. Clin Cancer Res 9: 295-306, 2003.

12. Lee YP, Schwarz EM, Davies M, Jo M, Gates J, Zhang X, Wu J and Lieberman JR: Use of zoledronate to treat osteoblastic versus osteolytic lesions in a severe-combined-immunodeficient mouse model. Cancer Res 62: 5564-5570, 2002.

13. Roodman GD: Mechanisms of bone metastasis. N Engl J Med 350: 1655-1664, 2004.

14. Nemeth JA, Harb JF, Barroso U Jr, He Z, Grignon DJ and Cher ML: Severe combined immunodeficient-hu model of human prostate cancer metastasis to human bone. Cancer Res 59: 1987-1993, 1999.

15. Kozlowski JM, Fidler IJ, Campbell D, Xu ZL, Kaighn ME and Hart IR: Metastatic behavior of human tumor cell lines grown in the nude mouse. Cancer Res 44: 3522-3529, 1984.
16. Jenkins DE, Yu SF, Hornig YS, Purchio T and Contag PR: In vivo monitoring of tumor relapse and metastasis using bioluminescent PC-3M-luc-C6 cells in murine models of human prostate cancer. Clin Exp Metastasis 20: 745-756, 2003.

17. Wang M and Stearns ME: Isolation and characterization of PC-3 human prostatic tumor sublines which preferentially metastasize to select organs in S.C.I.D. mice. Differentiation 48: 115-125, 1991.

18. Tsingotjidou AS, Zotalis G, Jackson KR, Sawyers C, Puzas JE, Hicks DG, Reiter R and Lieberman JR: Development of an animal model for prostate cancer cell metastasis to adult human bone. Anticancer Res 21: 971-978, 2001.

19. Haq M, Goltzman D, Tremblay G and Brodt P: Rat prostate adenocarcinoma cells disseminate to bone and adhere preferentially to bone marrow-derived endothelial cells. Cancer Res 52: 4613-4619, 1992. 